\title{
Evaluation of the topological characteristics of the turbulent flow in a 'box of turbulence' through 2D time-resolved particle image velocimetry
}

\author{
Huan Lian ${ }^{1} \cdot$ Nikolaos Soulopoulos $^{1} \cdot$ Yannis Hardalupas $^{1}$
}

Received: 30 March 2017 / Revised: 8 June 2017 / Accepted: 3 July 2017 / Published online: 3 August 2017

(C) The Author(s) 2017. This article is an open access publication

\begin{abstract}
The experimental evaluation of the topological characteristics of the turbulent flow in a 'box' of homogeneous and isotropic turbulence (HIT) with zero mean velocity is presented. This requires an initial evaluation of the effect of signal noise on measurement of velocity invariants. The joint probability distribution functions (pdfs) of experimentally evaluated, noise contaminated, velocity invariants have a different shape than the corresponding noise-free joint pdfs obtained from the DNS data of the Johns Hopkins University (JHU) open resource HIT database. A noise model, based on Gaussian and impulsive Salt and Pepper noise, is established and added artificially to the DNS velocity vector field of the JHU database. Digital filtering methods, based on Median and Wiener Filters, are chosen to eliminate the modeled noise source and their capacity to restore the joint pdfs of velocity invariants to that of the noise-free DNS data is examined. The remaining errors after filtering are quantified by evaluating the global mean velocity, turbulent kinetic energy and global turbulent homogeneity, assessed through the behavior of the ratio of the standard deviation of the velocity fluctuations in two directions, the energy spectrum of the velocity fluctuations and the eigenvalues of the rate-of-strain tensor. A method of data filtering, based on median filtered velocity using different median filter window size, is used to quantify the clustering of zero velocity points of the turbulent field using the radial distribution function (RDF) and Voronoï analysis to analyze the 2D time-resolved particle image velocimetry (TR-PIV) velocity measurements. It was
\end{abstract}

Yannis Hardalupas

y.hardalupas@imperial.ac.uk

1 Department of Mechanical Engineering, Imperial College London, London, UK found that a median filter with window size $3 \times 3$ vector spacing is the effective and efficient approach to eliminate the experimental noise from PIV measured velocity images to a satisfactory level and extract the statistical two-dimensional topological turbulent flow patterns.

\section{List of symbols}

$\tilde{A} \quad$ Reduced velocity gradient tensor

$f_{\mathrm{M}} \quad$ Intensity of the measured image

$f_{\mathrm{F}} \quad$ Intensity of the filtered image

$f \quad$ Intensity of the un-blurred noise-free image

$F_{\mathrm{M}} \quad$ Fourier form of the measured image

$F \quad$ Fourier form of the un-blurred noise-free image

$g \quad$ Optimal Wiener Filter

$G \quad$ Fourier form of the optimal Wiener Filter

$h \quad$ Point Spread Function of the optical system

$H \quad$ Fourier form of PSF of the degradation process

$H^{*}$ Conjugate of $H$

$n_{\mathrm{g}} \quad$ Local intensity of noise with Gaussian profile

$n_{\mathrm{sp}} \quad$ Local intensity of Salt and Pepper noise with Poisson distribution

$n \quad$ Intensity of the corrupting additive noise

$N \quad$ Fourier form of the corrupting additive noise

$\tilde{S} \quad$ Rate-of-strain tensor

$q^{2} \quad$ Turbulent kinetic energy

$R e_{\lambda} \quad$ Taylor scaled turbulent Reynolds number

$u_{i} \quad$ Turbulent flow velocity in $i$ direction

$x_{i} \quad$ Spatial displacement in $i$ direction

$\varepsilon \quad$ Turbulent kinetic energy dissipation rate

$\eta \quad$ Kolmogorov lengthscale

$\lambda \quad$ Taylor microscale

$\Gamma \quad$ Noise to signal ratio

$\tau_{\eta} \quad$ Kolmogorov time scale

$\Lambda \quad$ Integral lengthscale

$\tilde{\Lambda}_{i} \quad$ Eigenvalues of the rate-of-strain tensor 


\section{Introduction}

It has been observed that particle or droplet clustering occurs in two-phase flows due to the interaction of droplets or particles of different sizes with the turbulent eddies of the continuous phase flow. These clusters are regions where the dispersed phase has concentration higher than the average value and are complimented with regions of voids, where the dispersed phase concentration is lower than the average value. There are two proposed mechanisms to explain the formation of clusters of the dispersed phase. One is the centrifuging of particles by the turbulent eddies due to inertia (e.g., Maxey 1987; Wang and Maxey 1993; Eaton and Fessler 1994; Sundaram and Collins 1997; Hardalupas et al. 1990, 1992) and the second is a sweepstick mechanism, which suggests that the fluid acceleration field is swept by the local fluid velocity and particles tend to stick to and move with the zero acceleration points (e.g., Chen et al. 2006; Goto and Vassilicos 2006, 2008; Bragg et al. 2015). To be able to assess experimentally these two mechanisms for dispersed phase clustering, the topology of the flow structure in a turbulent flow must be detected and quantified across the different scales. This is challenging because measurements always contain noise from different sources, which influences the ability to analyze the flow structures across the different scales. Therefore, we would like to assess and optimize the ability to detect flow structures from experimental velocity measurements and this forms the purpose of this work.

To conceptually visualize coherent flow structures in turbulent flows, Perry and Chong (1987) described and classified the turbulent topological flow patterns using the critical points concept, where the slopes of the flow streamlines are indeterminate. These topological flow patterns are defined by invariants of the velocity gradient that can be quantified experimentally.

The calculation of velocity derivatives from two-dimensional and three-dimensional measurements of local velocity gradients with the discrete differentiation approach is largely affected by the spatial resolution of the measurement, the velocity vector spacing and the presence of 'noise' on the recorded images. Previous work, discussing the effect of spatial resolution on the fine scale turbulence, indicates that coarsely resolved velocity vector fields result in underestimation of the magnitude of velocity gradients and errors in the estimate of turbulent quantities (Antonia et al. 1994; Sugii et al. 2000; Lavoie et al. 2007; Worth et al. 2010). The three main sources of 'noise' on particle image velocimetry (PIV) images have been identified by Christensen (2004) as electronic noise of the camera, bias error due to pixel peak locking and gradient noise due to the local random velocity gradients within the field. Westerweel $(1997,2000)$ pointed out that the PIV measurement precision also depends on the sub-pixel displacement error in the processing algorithm. The influence of spatial resolution and noise on the measured velocity derivatives determines the accuracy of the estimation of the fine scale turbulent quantities and associated turbulent kinematic features, which, therefore, affect the understanding of the underlying physical processes. Thus, to obtain accurate flow visualization and interpretation of the physical process, there is a need to consider the influence of image noise on the determination of topological flow patterns defined using the phase portrait of the velocity invariants joint probability distribution functions (pdfs) (Perry and Chong 1987).

Buxton et al. (2011) studied the effect of spatial resolution and noise on the three-dimensional fine scale turbulent kinematic features and suggested that a minimum resolution of $2.5 \eta$ ( $\eta$ being the Kolmogorov scale of the flow turbulence) is required for the vector spacing. The shape of the joint pdfs of the velocity invariants $Q$ and $R$ is sensitive to the vector spacing, resolution and noise that the 'Vieillefosse tail' diminishes with decreased resolution or increased level of Gaussian noise. The definition of the velocity invariants $Q$ and $R$ is detailed in the work of Perry and Chong (1987). The reduced two-dimensional velocity invariants are defined in the current work.

The significance of the image noise is greater than that of the vector spacing resolution on the determination of the fine scale turbulent kinematic features. Cardesa et al. (2013) reported that the shape of the joint pdfs of the two-dimensional velocity invariants, measured by two-dimensional PIV, appears similar for various turbulent flow geometries and experimental flow conditions. In addition, a good agreement was observed between experiments and direct numerical simulations (DNS), so that joint pdfs and fine scale turbulent kinematic features, measured by planar PIV, is suggested to be less susceptible to noise and aliasing errors when compared to measurements with 3D diagnostics. However, the effect of image noise on the processed PIV velocity measurements requires examination (Khashehchi et al. 2010), which can be evaluated by adding artificial noise on the calculated flow velocity from DNS or LES flow computations (Okamoto et al. 2000; Atkinson et al. 2014).

The goal of the paper is to evaluate the experimental noise effects and select effective digital filtering techniques to extract the topological characteristics of the turbulent flow in a 'box of turbulence' through 2D time-resolved PIV. Different types and levels of noise are introduced to an openly available DNS data set. Then, the digital filtering techniques are optimized by evaluating their ability to remove the added noise from the DNS data while minimizing the introduced modifications to the characteristics of the reference DNS flow field. The optimum digital filtering techniques are then applied to the $2 \mathrm{D}$ time-resolved 
PIV measurements to obtain the best evaluation of the flow structures, which are relevant to the particle or droplet clustering in two-phase flows. The experimental setup is briefly described in Sect. 2. The facility of the 'box of turbulence' used in the current study that generates homogeneous and isotropic turbulence is summarized, while additional details are provided by Lian et al. (2013). The effect of image noise is evaluated in Sect. 3 by adding Gaussian noise on the DNS data from Johns Hopkins University (JHU) open resource database (Perlman et al. 2007; Li et al. 2008) of forced homogeneous and isotropic turbulence in a 10,243 periodic cube. The digital filtering methods are proposed and evaluated in Sect. 4. Three filtering approaches to eliminate the added image noise and spurious vectors are considered, namely (a) median filter (Westerweel 1994), (b) Wiener filter (Press et al. 1988) and (c) linear coupling of median filter and Wiener filter. The above methods are applied to measured 2D TR-PIV images of the flow velocity of homogeneous and isotropic turbulence. In addition to the evaluation of the filtering effect on the detection of turbulent flow topological patterns, the errors due to image filtering are also examined by evaluating the global mean velocity, turbulent kinetic energy, global turbulent flow homogeneity using the behavior of the ratio of the standard deviation of the velocity fluctuations in two directions, the energy spectrum of the velocity fluctuations and the eigenvalues of rate-of-strain tensor. Finally, Sect. 5 quantifies the effect of image noise on the clustering statistics of turbulent topological flow patterns, including instantaneous 'zero velocity' points. In this way, we establish the appropriate filtering approaches that eliminate the experimental noise to a satisfactory level to quantify the two-dimensional topological turbulent flow patterns in a 'box of turbulence'.

\section{Experimental setup}

The experimental velocity data are acquired from the flow in a 'box of turbulence' facility, which generates homogeneous and isotropic turbulence without mean flow. The operation principle of the 'box of turbulence' is similar to the facility of Hwang and Eaton (2004), Goepfert et al. (2009), Charalampous and Hardalupas (2010) and is described by Lian et al. (2013). Figure 1 shows a sketch of the facility, which comprised a cubic frame with 8 loudspeakers placed at the vertices, which are covered by a plate with 50 holes with $8 \mathrm{~mm}$ diameter. The loudspeakers operate at a random frequency between 40 and $60 \mathrm{~Hz}$ and generate arrays of synthetic jets, which all meet at the center of the cube. Careful fine-tuning of the amplitude of the imposed oscillations of the loudspeaker membranes allows balancing the flow and generating approximately a $40 \mathrm{~mm} \times 40 \mathrm{~mm} \times 40 \mathrm{~mm}$ cubic volume of homogeneous

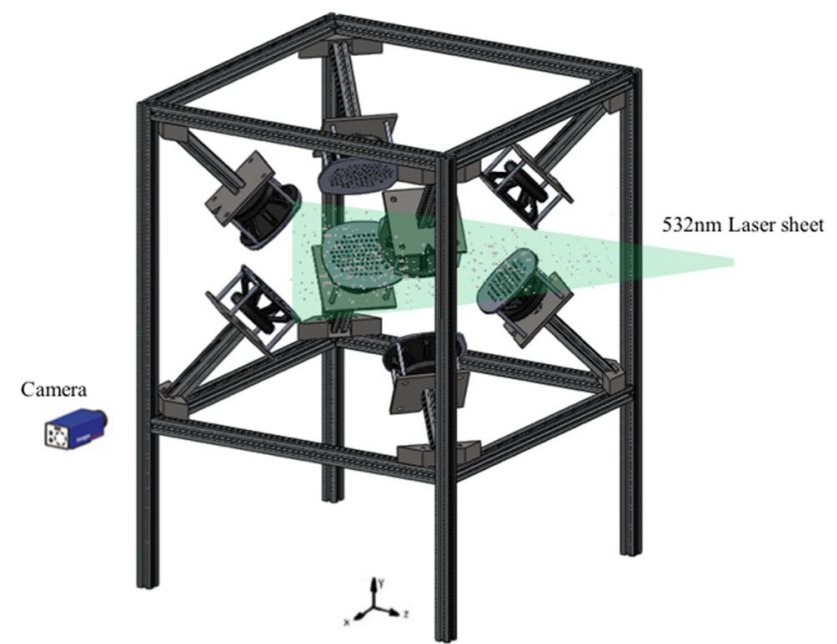

Fig. 1 Experimental Setup of the 'box of turbulence' facility for 2D TR-PIV measurement

and isotropic turbulence without mean flow at the center of the facility.

Particle image velocimetry (PIV) measured the instantaneous air flow characteristics after introducing fine glycol droplets with size less than 3 microns, generated by a VIVID stage fog generator. An Edgewave-IS series Nd: YAG laser operating at $532 \mathrm{~nm}$ was pulsed at high speed (up to $3 \mathrm{kHz}$ ), in order to illuminate a plane through the center of the 'box'. A planar laser sheet was shaped by a $-50 \mathrm{~mm}$ concave lens and a $500 \mathrm{~mm}$ convex lens with approximate thickness of $0.1 \mathrm{~mm}$ and was aligned at the center of an illuminated area of interest (AOI) of around $40 \times 40 \mathrm{~mm}^{2}$ at the 'box' center. The laser sheet illuminated the 'seeding' particles and the intensity of the scattered light was recorded by a Photron APX CMOS camera $(1024 \times 1024$ pixel) using a $105 \mathrm{~mm}$ lens $\mathrm{f} / 2.8$, leading to a linear magnification of 0.3 and resulting in spatial resolution of $45.5 \mu \mathrm{m} / \mathrm{pixel}$. The rate of the reported measurements is set at $1500 \mathrm{~Hz}$. The TR-PIV images were processed with Davis 7.2 software from Lavision $\mathrm{GmbH}$. The first pass interrogation window size was $64 \times 64$ pixels with $50 \%$ overlap and the second pass was $16 \times 16$ pixels with $50 \%$ overlap, resulting in velocity vector spacing of 8 pixels, which represents absolute distance of $364 \mu \mathrm{m}$. Since the Kolmogorov scale $\eta$ is $167 \mu \mathrm{m}$ for the presented condition of turbulent Reynolds number of 147 (Lian 2014), the velocity vector spacing is smaller than $2.5 \eta$, as suggested by Worth et al. (2010) for appropriate measurement precision of velocity derivatives. A $3 \times 3$ pixels Gaussian kernel smoothing was applied to the images during the multi-pass, with no further post processing.

The Kolmogorov scale and the turbulent Reynolds number are estimated according to the equations of: 
$\operatorname{Re}_{\lambda}=\frac{\lambda\left(q^{2} / 3\right)^{1 / 2}}{v}$,

where $\nu$ is the kinematic viscosity of the air, which is $1.57 \times 10^{-5} \mathrm{~m}^{2} / \mathrm{s}$ at atmospheric pressure and temperature, and $\lambda$ is the Taylor length scale defined as

$\lambda=\left(\frac{5 v q^{2}}{\varepsilon}\right)^{1 / 2}$,

where the turbulent kinetic energy $q^{2}$ is defined as

$q^{2}=3 \frac{u_{1, \mathrm{rms}}^{2}+u_{2, \mathrm{rms}}^{2}}{2}$.

The details of how the kinetic energy $q^{2}$, Taylor microscale $\lambda$, Kolmogorov timescale $\tau_{\eta}$, Kolmogorov lengthscale $\eta$, integral lengthscale $\Lambda$ are evaluated from the velocity measurements are described in George (2013) and Lian et al. (2013) and are not repeated here.

The energy dissipation rate was estimated following Eq. (4), under the assumption of global isotropy (Cardesa et al. 2013). The velocity gradient was calculated using the central difference algorithm.

$$
\begin{aligned}
\varepsilon= & \frac{5}{2}\left\langle\left(\frac{\partial u_{1}}{\partial x_{1}}\right)^{2}\right\rangle+\frac{5}{2}\left\langle\left(\frac{\partial u_{2}}{\partial x_{1}}\right)^{2}\right\rangle \\
& +\frac{5}{2}\left\langle\left(\frac{\partial u_{1}}{\partial x_{2}}\right)^{2}\right\rangle+\frac{5}{2}\left\langle\left(\frac{\partial u_{2}}{\partial x_{2}}\right)^{2}\right\rangle .
\end{aligned}
$$

The DNS data set is used to identify the effect of the primary experimental noise types on the velocity tensor invariants and principle components, which is expected to be independent of the turbulent conditions of the current experiment. The joint pdfs of the velocity invariants and the pdfs of the eigenvalues of the rate-of-strain tensor detailed in the following sections are normalized to ensure independence from the absolute values of the different flow conditions. In this way, this evaluation through the DNS data set can optimize the digital filtering techniques that can then be used for the processing of the experimental data. We chose to use the open source JHU DNS database, so that the results reported in the paper can be easily repeated,

A summary of the absolute values of the experimental turbulent conditions and the forced isotropic turbulence calculated from the DNS of JHU open resource database is shown in Table 1 for reference. However, as explained above, the normalized DNS data are used to optimize the filtering techniques for the processing of the experimental data.

\section{Noise model on turbulent topological flow pattern and rate-of-strain tensor}

\subsection{Turbulent topological flow pattern}

The classification of the topological turbulent flow patterns is briefly described below, including definitions of two-dimensional velocity gradients, velocity invariants $p$ and $q$ and the representation of the invariant phase portrait. Detailed descriptions on this topic can be found in Perry and Chong (1987) and Cardesa et al. (2013). It should be noted that a two-dimensional analysis is applied to both the DNS data set and the experimental images.

The reduced two-dimensional velocity gradient tensor $\tilde{A}$ is a first-order Cartesian tensor defined as:

$\tilde{A}=\left[\begin{array}{ll}\frac{\partial u_{1}}{\partial x_{1}} & \frac{\partial u_{1}}{\partial x_{2}} \\ \frac{\partial u_{2}}{\partial x_{1}} & \frac{\partial u_{2}}{\partial x_{2}}\end{array}\right]$.

The characteristic polynomial equation of $\tilde{A}$ is derived as follows.

$\alpha^{2}+p \alpha+q=0$,

where

$p=-\operatorname{tr}(\tilde{A})=-\left(\begin{array}{ll}\frac{\partial u_{1}}{\partial x_{1}} & \frac{\partial u_{2}}{\partial x_{2}}\end{array}\right)$

$q=-\operatorname{det}(\tilde{A})=\frac{\partial u_{1}}{\partial x_{1}} \frac{\partial u_{2}}{\partial x_{2}}-\frac{\partial u_{1}}{\partial x_{2}} \frac{\partial u_{2}}{\partial x_{1}}$

The eigenvalues of $\tilde{A}$ can be calculated by solving the characteristic polynomial equation, so that they are uniquely determined by the characteristic polynomial coefficients $p$ and $q$. The classifications of the local critical points are determined by the local eigenvalue of $\tilde{A}$. As a consequence, the categories of the critical points depend entirely on the values of the characteristic polynomial coefficients $p$ and $q$. The
Table 1 Conditions of homogeneous, isotropic turbulence for experiments and DNS calculations

\begin{tabular}{llllllll}
\hline Turbulent quantities & $q^{2}\left(\mathrm{~m}^{2} / \mathrm{s}^{2}\right)$ & $\varepsilon\left(\mathrm{m}^{2} / \mathrm{s}^{2}\right)$ & $R e_{\lambda}$ & $\lambda(\mathrm{m})$ & $\tau_{\eta}(\mathrm{s})$ & $\eta(\mathrm{mm})$ & $\Lambda(\mathrm{m})$ \\
\hline Experiment & 2.148 & 19.69 & 147 & 0.003 & 0.001 & 0.134 & 0.032 \\
JHU DNS & 0.695 & 0.093 & 433 & 0.118 & 0.045 & 2.87 & 1.376 \\
\hline
\end{tabular}




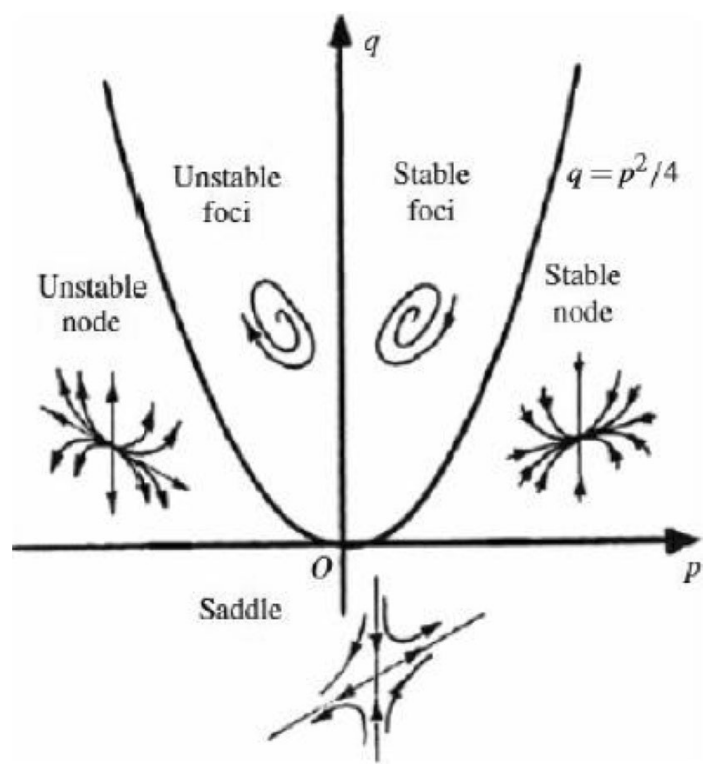

Fig. 2 The topological categories of the critical points in a twodimensional velocity field (Perry and Chong 1987)

topological categories of the critical points in a two-dimensional velocity field were shown in a form of a phase portrait of the characteristic polynomial coefficients $p$ and $q$ (Perry and Chong 1987). A reproduction of the Perry and Chong (1987) phase portrait is presented in Fig. 2.

Five different topological categories of the critical points are defined in the phase portrait.

1. Unstable node if $p<0$ and $q>0$ and $q<p^{2} / 4$;

2. unstable focus if $p<0$ and $q>0$ and $q>p^{2} / 4$;

3. stable node if $p>0$ and $q>0$ and $q<p^{2} / 4$;

4. stable focus if $p>0$ and $q>0$ and $q>p^{2} / 4$;

5. saddle points if $p<0$ and $q<0$.

Since the topological turbulent flow patterns are defined from the joint pdfs phase portrait of the velocity invariants, the noise model below is developed based on the phase portrait.

\subsection{Rate-of-strain tensor}

The velocity gradient tensor $A_{i j}$ can be expressed as the summation of a symmetric and an asymmetric component:

$A_{i j}=\frac{\partial u_{i}}{\partial x_{j}}=S_{i j}+\Omega_{i j}$

where $S_{i j}$ is the rate-of-strain tensor and $\Omega_{i j}$ is the rate-ofrotation tensor, respectively. The rate-of-strain tensor has been extensively used to study flow dynamics in various flow configurations. In the current paper, the rate of strain is used to evaluate the global turbulent homogeneity for the purpose of quantifying noise propagation and filtering errors.

The eigenvalues of the two-dimensional rate-of-strain tensor $\tilde{S}$ are given as

$$
\begin{aligned}
\tilde{S}= & {\left[\begin{array}{cc}
\frac{\partial u_{1}}{\partial x_{1}} & \left(\frac{\partial u_{1}}{\partial x_{2}}+\frac{\partial u_{2}}{\partial x_{1}}\right) / 2 \\
\left(\frac{\partial u_{1}}{\partial x_{2}}+\frac{\partial u_{2}}{\partial x_{1}}\right) / 2 & \frac{\partial u_{2}}{\partial x_{2}}
\end{array}\right] } \\
\tilde{\Lambda}= & \left(\frac{\partial u_{1}}{\partial x_{1}}+\frac{\partial u_{2}}{\partial x_{2}}\right) / 2 \pm \frac{1}{2} \sqrt{\left(\frac{\partial u_{1}}{\partial x_{1}}+\frac{\partial u_{2}}{\partial x_{2}}\right)^{2}} \\
& -4\left(\frac{\partial u_{1}}{\partial x_{1}} \cdot \frac{\partial u_{2}}{\partial x_{2}}-\left(\left(\frac{\partial u_{1}}{\partial x_{1}}+\frac{\partial u_{2}}{\partial x_{2}}\right) / 2\right)^{2}\right) .
\end{aligned}
$$

Statistical results from Eq. (11) can be derived with the averages of $\tilde{\Lambda}_{1}$ and $\tilde{\Lambda}_{2}$ equal in magnitude and opposite in sign (Cardesa et al. 2013), which yields

$\left\langle\tilde{\Lambda}_{1}\right\rangle=-\left\langle\tilde{\Lambda}_{2}\right\rangle$.

The ratio of the eigenvalues is used to quantify the homogeneity of the turbulent flow for evaluating the 'noise' propagation and filtering errors.

\section{3 'Noise' model}

The concept and theory of digital noise transfer function (point spread function PSF), defined and developed in image processing community, is briefed below to provide foundations of 'noise' elimination in the PIV measured velocity vector fields.

The noise present in a digital image is usually modeled as either impulse noise or Gaussian noise. The impulse noise is also generally referred to as 'Salt and Pepper noise' and assumed to be Poisson distributed according to the form:

$p(k)=\frac{e^{-n_{p x}} n_{p x}^{k}}{k !}$.

Given a specific window size, the probability of having $k$ pixels affected by noise yields $p(k)$, where $n_{p x}$ is the average number of affected pixels and represents the variance of the Poisson distribution. The 'Salt and Pepper noise' tends to have a local effect on individual pixels.

The Gaussian noise is associated with the random noise value at a given pixel, which is drawn from a Gaussian distribution with given expectation and variance, and affects the intensity value of each pixel in the whole image area. The random noise field from these two noise sources is 
added to the 'true' pixel intensity value, thus referred to as additive noise.

Apart from the noise presented in a digital image, the blurring of an optical system is measured by the Point Spread Function (PSF), which expresses how much the input intensity value affects the output intensity value over locations of pixels when imaging a point source (Petrou and Petrou 1999). The line spread function (LSF) is the PSF along a given direction and could be measured by the scanning edge-knife technique (Soulopoulos et al. 2014) as the first order derivative of the edge spread function (ESF) or estimated according to a Gaussian profile when the image intensifier is not employed in the optical system. The Fourier transform of the line spread function is an important parameter in the process of digital filtering and is generally referred to as the modulation transfer function (MTF).

Therefore, a measured digital image is blurred by the PSF and superimposed additive noise. The combination of these effects is modeled as 'Salt and Pepper noise' or Gaussian noise or a combination of these two noise sources. The imposed bias on measured velocity fields has been summarized by Christensen (2004) and Westerweel (2000), who demonstrated the complicated effects of digital image noise and PIV processing algorithms. A noise model can be developed for the evaluation of the topological characteristics of turbulent flows and the fine scale turbulent kinematics to guide the development of the noise elimination techniques.

To establish the noise model and observe the influence of noise on the topological flow patterns and the fine scale turbulent kinematics, the two defined noise sources in the community of image processing, Gaussian noise and Salt and Pepper noise, have been added to the 'un-blurred' 'noise-free' two-dimensional velocity vector fields acquired from the DNS data of Johns Hopkins University (JHU) open resource turbulence database (Perlman et al. 2007; Li et al. 2008) expressed as:

$\vec{u}_{i, p}(x, y)=\vec{u}_{i, D N S}(x, y)+n_{\mathrm{g}}(x, y)+n_{\mathrm{sp}}(x, y)$,

where $\vec{u}_{i, p}(x, y)$ is the pseudo velocity component with artificial additive noise at location $(x, y) ; \vec{u}_{i, D N S}(x, y)$ is the local velocity component from the DNS database of JHU; $n_{\mathrm{g}}(x, y)$ is the local intensity of noise with Gaussian profile; $n_{\mathrm{sp}}(x, y)$, is the local intensity of Salt and Pepper noise with Poisson distribution.

Zero mean Gaussian noise with variance of 0.001 and 0.005 and 'Salt and Pepper noise' with density of 0.001 and 0.005 have been separately added with the other term set as zero, corresponding to value of $10^{-3}$ times the root mean square (rms) of the turbulent flow velocity fluctuations $u_{\mathrm{rms}}=3.5 \mathrm{~m} / \mathrm{s}$, the same levels as those used by Buxton et al. (2011) for the study of the effect of noise

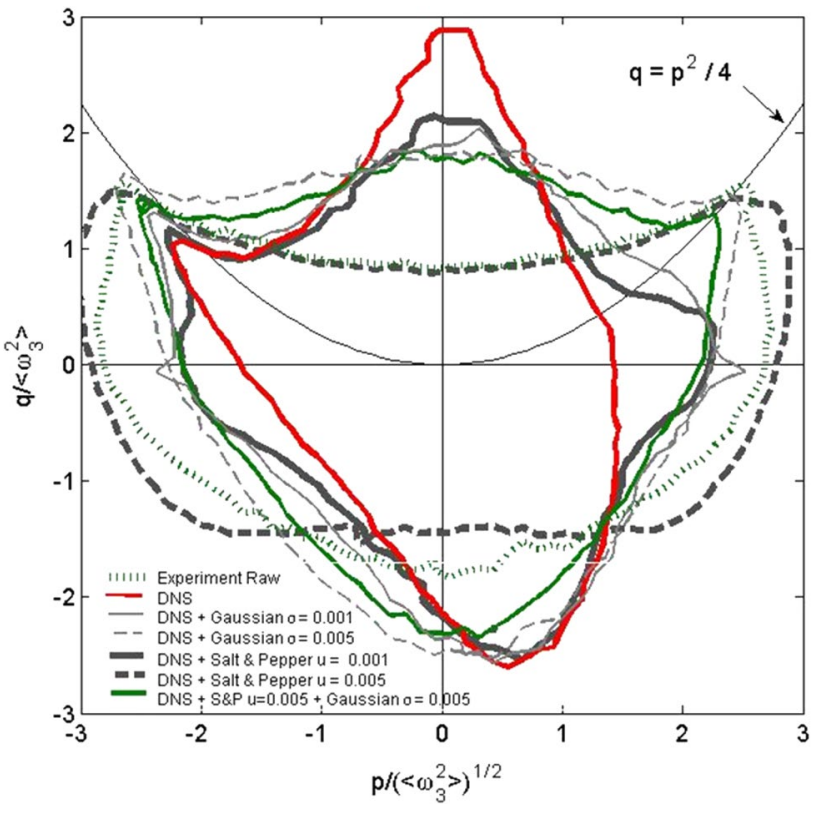

Fig. 3 The joint pdfs of velocity invariants of homogeneous, isotropic turbulence as estimated from the JHU DNS simulation with added Gaussian noise and/or Salt and Pepper noise and experimentally measured without applying any noise reduction method during image processing

on three-dimensional conditions. Linear combination of the Gaussian noise with variance of 0.005 and the Salt and Pepper noise density of 0.005 has also been tested. The resulting joint pdfs of velocity invariants are shown in Fig. 3.

Figure 3 shows that the addition of different levels of zero mean Gaussian noise modifies significantly the shape of the joint pdfs of the velocity invariants. The tails of the pdfs are more pronounced for higher magnitude of Gaussian noise and the shape expands along the horizontal axis. The skewness of the joint pdf, calculated from the DNS, is reduced when Gaussian noise is artificially added. The addition of Salt and Pepper noise modifies the shape of the joint pdfs greatly, when the magnitude of density is 0.005 . The skewness could no longer be observed and the pointy edges are rounded as shown by the thick dash line in Fig. 3.

The effect of both Gaussian and Salt and Pepper noise on the joint pdfs is tested by linearly adding up the two noise sources, namely the Gaussian noise with zero mean and variance of 0.005 and the Salt and Pepper noise with density of 0.005 . It is shown by the green solid line in Fig. 3 that the deterioration of the pdfs due to Salt and Pepper noise is reduced after introducing the Gaussian noise. The joint pdfs of the velocity invariants, calculated from the raw experimental data, are shown by the dotted thick green line. It is indicated that the joint pdfs of the raw 
experimental data are in transition between the joint pdfs of the combined Gaussian and Salt and Pepper noise and the Salt and Pepper with density level of 0.005 , suggesting that the Salt and Pepper noise is dominant in the velocity vector fields measured by PIV.
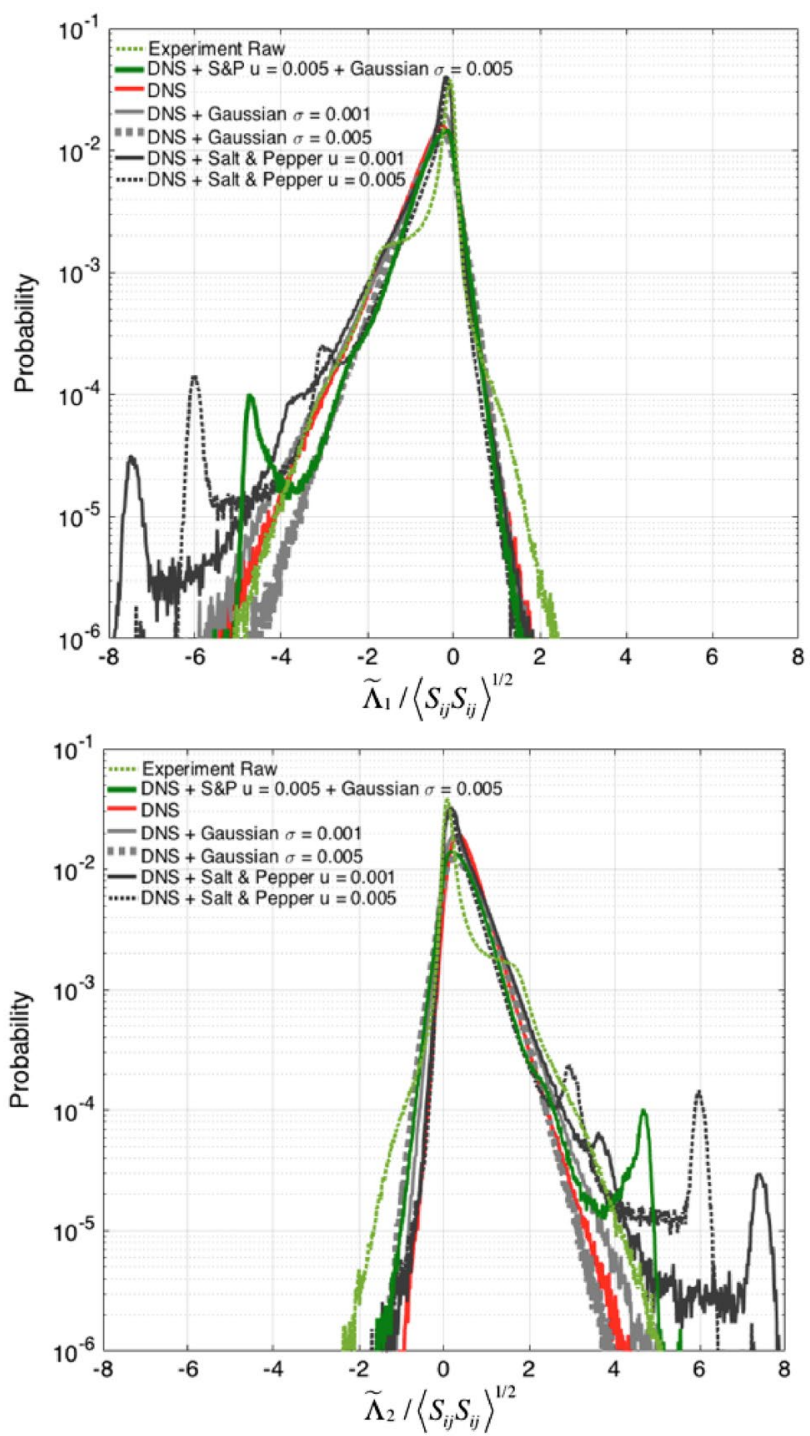

Fig. 4 The pdfs of eigenvalues of rate-of-strain tensor of homogeneous, isotropic turbulence as estimated from JHU DNS simulation with added Gaussian noise and Salt and Pepper noise and experimentally measured without applying any noise reduction method during image processing
To evaluate the effect of noise on the rate-of-strain tensor, the resulting pdfs of the eigenvalues of the rate-of-strain tensor, calculated following Eq. (11), are shown in Fig. 4. The two eigenvalues are plotted in separate figures to avoid superimposition.

The addition of different levels of zero mean Gaussian noise slightly modifies the shape of the pdfs of the eigenvalues of rate-of-strain tensor. The pdfs of the eigenvalues are significantly affected by the addition of different levels of Salt and Pepper type noise. The skewness of the pdfs in the high probability region (i.e. higher than $10^{-2}$ ) caused by the addition of Salt and Pepper type of noise matches the experimental trend, shown by the light green line. However, in the region with probability of $10^{-3}$ to $10^{-2}$, the experimentally measured eigenvalues are closer to zero in comparison to the simulation results. Also, for the normalized eigenvalues around $-8,-6$ and 6, 8, the addition of Salt and Pepper noise causes local saturation in probability between $10^{-6}$ and $10^{-4}$.

The eigenvalues resulted from the coupled zero mean Gaussian noise with variance of 0.005 and Salt and Pepper noise with density of 0.005 recover towards DNS simulation comparing to Salt and Pepper noise, shown by the dark green solid line in Fig. 4. This finding for the pdfs of the eigenvalue is consistent with the finding for the joint pdfs of the velocity invariant that the corruption due to Salt and Pepper noise is reduced after introducing the Gaussian noise. Also, the linear summation of the two noise sources could not re-construct the experimentally measured eigenvalues with probability of $10^{-3}$ to $10^{-2}$, suggesting that the interaction of the two types of noise could be non-linear.

In contrary to the velocity invariants, the experimentally measured eigenvalues are more similar to those obtained after the addition of Gaussian noise. This indicates that the effect of Gaussian noise might be more pronounced than that of the Salt and Pepper noise in estimating eigenvalues of rate-ofstrain tensor, while the Salt and Pepper noise dominates the measurement of the velocity invariants.

The ratio of the average of the two eigenvalues that represents local homogeneity of the turbulent flow is shown in Table 2. The addition of different type and level of noise does not change the ratio of eigenvalues, suggesting that the global homogeneity is not affected by the noise addition. This is expected, since the noise addition is uniform spatially.

In summary, a noise model was established for the PIV measured velocity data, which has the ability to mimic the effect of experimental noise on the topological

Table 2 The ratio of the average of the two eigenvalues of rate-of-strain

\begin{tabular}{|c|c|c|c|c|c|c|}
\hline $\begin{array}{l}\text { Ratio of the two } \\
\text { eigenvalues }\end{array}$ & DNS & $\begin{array}{l}\text { DNS + Gaussian } \\
0.001\end{array}$ & $\begin{array}{l}\text { DNS + Gaussian } \\
0.005\end{array}$ & $\begin{array}{l}\text { DNS }+S \text { and } P \\
0.001\end{array}$ & $\begin{array}{l}\mathrm{DNS}+\mathrm{S} \text { and } \mathrm{P} \\
0.005\end{array}$ & $\begin{array}{l}\text { DNS + S and P } 0.005 \\
+ \text { Gaussian } 0.005\end{array}$ \\
\hline$\left\langle\tilde{\Lambda}_{1}\right\rangle /\left\langle\tilde{\Lambda}_{2}\right\rangle$ & -1.00 & -1.00 & -1.00 & -1.00 & -1.00 & -1.00 \\
\hline
\end{tabular}


characteristics of a turbulent flow and fine scale turbulent kinematics, namely the joint pdfs of velocity invariants and the pdfs of the eigenvalues of rate-of-strain tensor. The topological turbulent flow patterns, defined by the phase portrait of the joint pdfs, are sensitive to both the Gaussian noise and Salt and Pepper noise, while the latter effect is more pronounced. The eigenvalues of rate-of-strain tensor are also sensitive to both types of noise; however, the Gaussian noise appears to be more critical. The finding on the dominating noise type is then used to provide guidance for the development of digital filtering techniques for noise elimination from PIV velocity data to evaluate the topological turbulent flow patterns as well as the rate-of-strain tensor. The following section describes the developed digital filtering techniques.

\section{Digital filtering techniques}

For tensor invariants and its principle components, we identified the Gaussian noise and the impulsive Salt and Pepper noise as the two primary experimental noise types. For the presented flow data, the tensor invariants seem to be more sensitive to the Salt and Pepper while the principal components to Gaussian. For the impulsive Salt and Pepper noise, the standard median filter is one of the most popular non-linear filters used due to its good de-noising power and computational efficiency (Huang et al. 1979). Other types of non-linear filters, i.e., adaptive median filter, cascade and recursive filters, are applicable but more suitable for high noise intensity level $>50 \%$. In our case, the noise intensity is considered low; thus, the standard median filter was chosen in the current work, which forces the distinct intensity to be more like its neighbors and eliminates isolated velocity spikes (Westerweel 1994). Digital filters that smooth the velocity field, including convolution or wavelet based filters and Wiener filters, are generally used to eliminate the Gaussian noise (Petrou and Petrou 1999). Among these digital filters, Wiener filter is computationally efficient and the most important advantage is that it does not requires parameter adjustment except estimation of the noise to signal ratio. Other filtering methods, for instance the Gaussian filter, involve filter width and parameter optimization strongly dependent on the noise level (Vétel et al. 2011). Thus, we chose the Wiener filter to eliminate the Gaussian noise in the current work.

This section presents the theory of the digital filtering behind the Wiener filter and the Median filter approaches in order to enhance and restore noisy images with the application of these digital filtering techniques on the two-dimensional velocity vector field.

\subsection{The Wiener filter and the median filter}

The Wiener filter is a technique to restore the image blurred by PSF and mainly the Gaussian additive noise. The Median filter is an effective method to remove impulsive Salt and Pepper noise.

The theory of the Wiener filter is briefed first. In the following discussion, it should be noted that lower case expressions donate image intensity values in physical space and upper case expressions donate image intensity values in Fourier space. An image blurred by PSF and with additive noise can be expressed as

$f_{\mathrm{M}}(r)=\int_{-\infty}^{+\infty} \int_{-\infty}^{+\infty} h\left(r-r^{\prime}\right) f\left(r^{\prime}\right) d r^{\prime}+n(r)$,

where $f_{\mathrm{M}}(r)$ is the measured image, $f(r)$ is the un-blurred noise-free image, $h(r)$ is the PSF of the optical system and $n(r)$ is the corrupting additive noise. The Fourier form of this expression is:

$F_{\mathrm{M}}(k)=H(k) F(k)+N(k)$,

Applying a digital filter, the filtered image can be expressed as:

$f_{\mathrm{F}}(r)=\int_{-\infty}^{+\infty} f_{\mathrm{M}}\left(r^{\prime}\right) g\left(r-r^{\prime}\right) \mathrm{d} r^{\prime}$

with its Fourier form as:

$F_{\mathrm{F}}(k)=F_{\mathrm{M}}(k) G(k)$.

The least square error between the un-blurred noise-free image and the filtered image is defined as:

$\mathrm{e}^{2}=E\left\{\left[f(r)-f_{\mathrm{F}}(r)\right]^{2}\right\}$,

where $E$ donates the expectation.

The Wiener filter is an optimal linear solution to the least squares error estimation between the ideal image and the filtered image. Its Fourier form has been obtained (Petrou and Petrou 1999) as:

$G(k)=\frac{H^{*}(k)}{|H(k)|^{2}+\frac{S_{n}(k)}{S(k)}}$,

where $G(k)$ is the Fourier form of an optimal Wiener filter, $H(k)$ is the Fourier form of PSF of the degradation process, $H^{*}(k)$ is the conjugate of $H(k)$. The spectral densities of the additive noise and un-blurred noise-free image are $S_{n}(k)$ and $S(k)$.

The construction of the Wiener Filter requires estimation or measurement of the PSF. In the current work, the PSF is represented by a Gaussian profile, which is expected for most of the optical systems without the presence of an image intensifier that alters the PSF with a longer tail. 
In the context of fluid mechanics, where the Wiener Filter is applied to the two-dimensional velocity vector field, the estimation of spectral density of noise and un-blurred noise-free 'image' is achieved via kinetic energy power spectrum following the approach of Soulopoulos et al. (2014). The kinetic energy power spectrum of the turbulent flow condition, detailed in Table 1, is shown in Fig. 5.

According to the procedure described by Soulopoulos et al. (2014), power spectral density estimation is shown in Fig. 5. $F_{\mathrm{M}}(k)$ represents the measured kinetic energy power spectrum of the turbulent flow with Reynolds number of $147 . N(k)$ is the estimated noise spectrum using cubic spline extrapolation from the measured power spectrum. The filtered energy spectrum is the deduction between the two, shown as $F_{\mathrm{M}}(k)-N(k)$. The term $S_{n}(k) / S(k)$ of Eq. (20) can be simplified as a constant $\Gamma$, following suggestions by Petrou and Petrou (1999), which indicates the noise to signal ratio. In the current work, $\Gamma$ is approximated as $1 / 10$.

The procedure of applying Wiener filter on the twodimensional velocity vector field is summarized as follows:

1. Evaluate the modulation transfer function (MTF) $H(k)$, which is the Fourier transform of the point spread function (PSF). The PSF can be approximated as a Gaussian profile or measured with the scanning knife-edge technique.

2. Estimate the constant $\Gamma$ from the kinetic energy power spectrum.

3. Calculate the two-dimensional Fourier Transform of the instantaneous velocity vector field measured by PIV, $F(k)$.

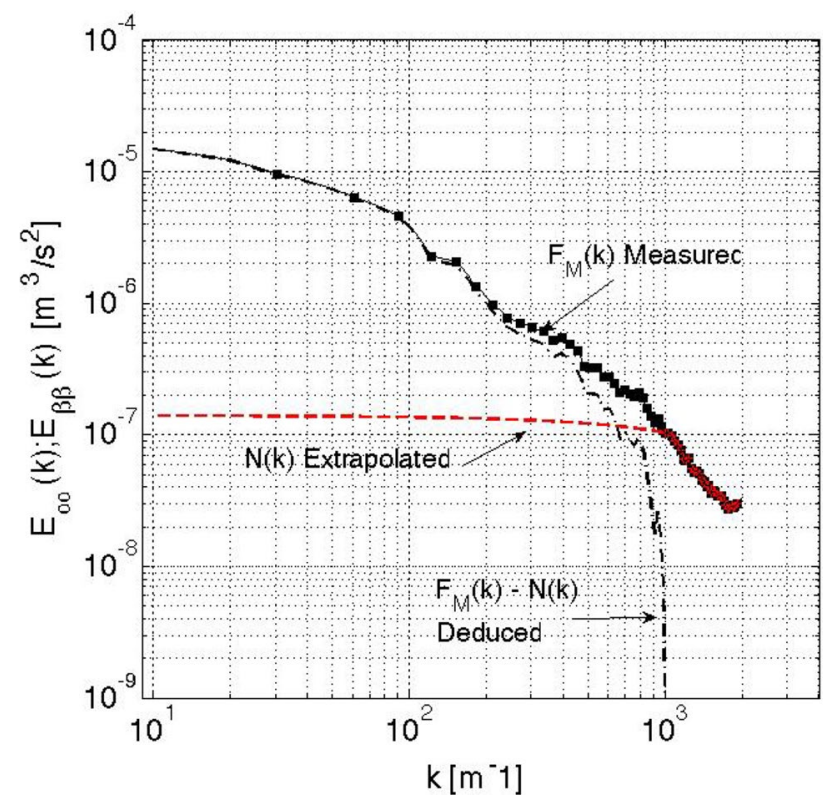

Fig. 5 Power spectral density estimation with optimal Wiener filter
4. Multiply $F(k)$ point by point with the constructed Wiener filter $G(k)$.

5. Calculate the inverse Fourier transform to obtain the filtered instantaneous velocity vector field, $F_{\mathrm{f}}(r)$.

6. Calculate the fluctuating velocity vector field from the filtered instantaneous velocity vector field for velocity gradient and invariants estimation.

The Median filter is a non-linear digital de-noising method widely used by the image processing community to remove the impulsive Salt and Pepper noise, which, according to Westerweel (1994), represents similar properties as that of spurious velocity vectors occurring in PIV measurements. It replaces the current pixel entry with the median value of the neighboring pixel entries within the square filter window size of $n \times n$, where $n$ is usually set as an integer less than 10 .

\subsection{Filtering results}

The raw experimental two-dimensional velocity vector fields are processed with digital filters constructed from a Wiener filter and a median filter.

The resulting joint pdfs of velocity invariants are plotted in Fig. 6 with thick black dashed line. The shape of the joint pdfs is similar to that of the DNS data with Salt and Pepper noise of density level 0.005 , suggesting that the Wiener Filter removes certain levels of Gaussian noise,

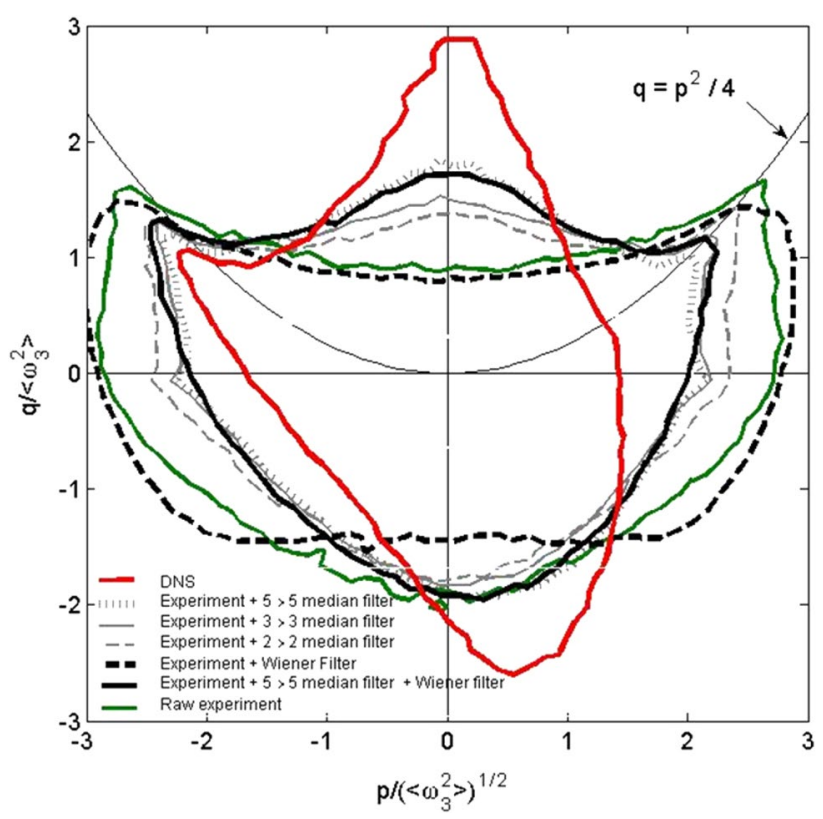

Fig. 6 The joint pdfs of velocity invariants of homogeneous, isotropic turbulence from experiment, processed by median filter, Wiener filter and linear coupling of median and Wiener filters 
leaving the velocity vector fields dominated by Salt and Pepper noise. The median filter with different window sizes $(2 \times 2,3 \times 3$ and $5 \times 5$ vector spacing $)$ has been applied to the experimental PIV data, as shown in Fig. 6. The $p$ and $q$ joint pdfs shrink and approach the 'ideal shape', identified by the DNS data without any noise, better with a larger window size. It is observed that the median filter effectively removes the spurious velocity vectors.

As indicated by the noise model established in the previous section, the velocity vector fields are contaminated by both Gaussian and Salt and Pepper noise with the latter dominating. The combined median and Wiener filters seem to be the solution to filter the experimental velocity vector fields thus tested and shown in Fig. 6. No significant improvement, but only a bit of smoothing, is observed comparing to the result from filtering with a $5 \times 5$ vector spacing window sized Median Filter. It should be noted that the sequence of applying the Wiener and Median Filters on the images does not modify the result. This confirms the suggestion that the Salt and Pepper noise, effectively the spurious vector in the velocity vector fields, is the dominant factor in the noise model influencing the evaluation of the topological characteristics of the turbulent flow.

The experimentally measured pdfs of the eigenvalues deviate from the pdfs estimated from the DNS database. Following the same filtering approach applied to velocity invariants, the filtered pdfs of the eigenvalues of the rateof-strain tensor are shown in Fig. 7.

The median filter with different window sizes $(2 \times 2$, $3 \times 3$ and $5 \times 5$ vector spacing) has been applied to the experimental PIV data, shown as gray lines in Fig. 7. The green raw result has a 'dip' at probability of $10^{-2}$ to $10^{-3}$. The median filter effectively removes local velocity vectors with high variance at the range of high probability, yet cause deviation at lower range of probability with the shapes of the pdfs of the eigenvalues deviate further away from the DNS results, suggesting the eigenvalues are sensitive to the fine modifications on the local velocity fields.

The resulting pdfs of the eigenvalues from the twodimensional raw experimental velocity vector fields processed with the constructed Wiener Filter are plotted as the thick black dashed lines in Fig. 7. The Wiener Filter removes certain levels of Gaussian noise leaving the velocity vector fields dominated by Salt and Pepper noise. A significant improvement is observed relative to the result following filtering by a $5 \times 5$ vector spacing window Median Filter. Furthermore, the combined Wiener filter and Median filter with window size of $5 \times 5$ vector spacing yields the best filtering result. This confirms the finding of the previous section that the Gaussian noise is the dominant factor influencing the measurement of the rate-of-strain tensor.

In summary, the Gaussian noise is the dominant factor influencing the measurement of the rate-of-strain tensor.
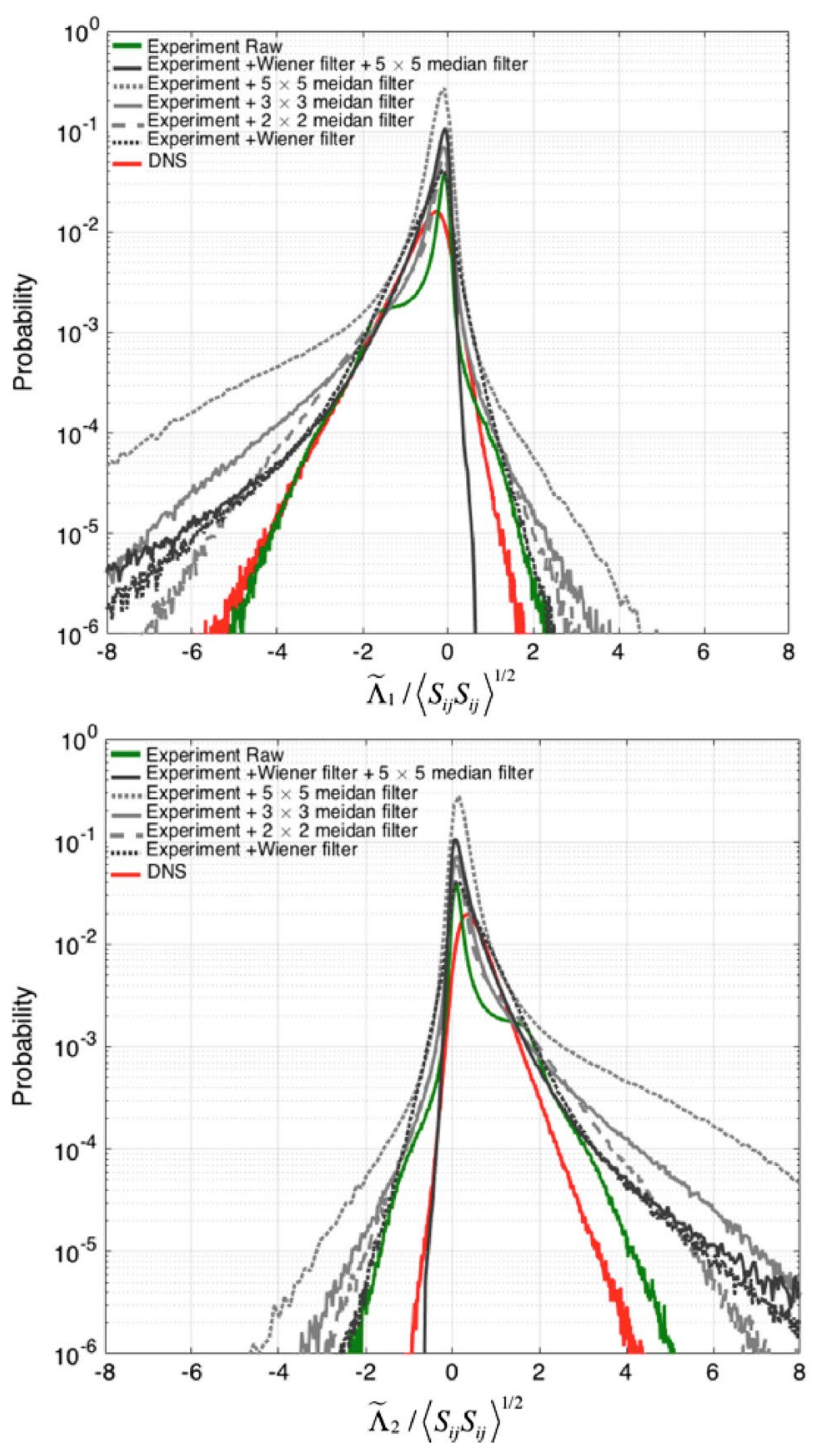

Fig. 7 The pdfs of eigenvalue of rate-of-strain tensor of homogeneous, isotropic turbulence from experiment, processed by median filter, Wiener filter and coupled median and Wiener filters

However, the Salt and Pepper noise, effectively the spurious vector in velocity vector fields, is the dominant factor in the noise model influencing the evaluation of the topological characteristics of the turbulent flow. The proposed digital filtering techniques recover the rate-of-strain tensor and topological characteristics derived from velocity invariants. The filtering errors are quantified in the following Section in order to evaluate the modifications to turbulent statistics introduced by the digital filtering process.

\subsection{Filter error estimation}

The velocity vector field restored by the Wiener filter with noise to signal ratio set at $\Gamma=1 / 10$ is used as the 
benchmark, because the optimal Wiener filter minimizes the error relative to the un-blurred, noise-free image (Vétel et al. 2011) and demonstrates good accuracy in temporal filtering (Oxlade et al. 2012). The filtering errors with other filtering techniques are examined by comparing with the benchmark calculation of the global mean velocity, turbulent kinetic energy and the global turbulent homogeneity, which is evaluated by the behavior of the energy spectrum and the eigenvalues of the rate-of-strain tensor.

The turbulent spectrum calculated from the two-point velocity correlation function processed via different filtering techniques is shown in Fig. 8. The red dotted line is the benchmark spectrum processed with a Wiener filter. Although the Wiener filter is optimal in the sense of error minimization, it introduces negative bias at the high frequency range of the energy spectrum. As observed in the rms of the velocity fluctuations, the median filter decreases

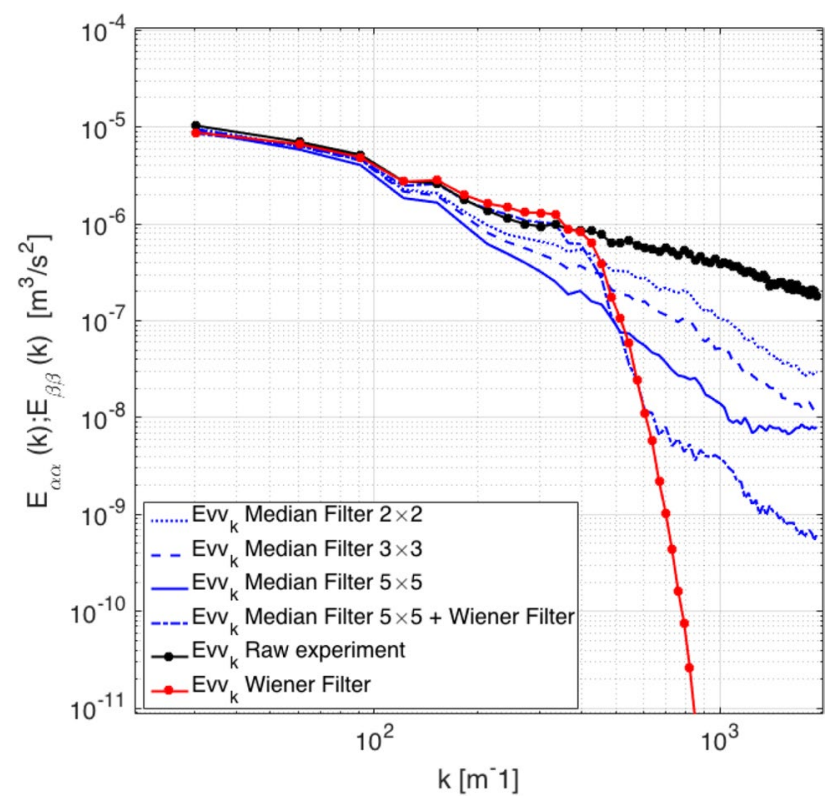

Fig. 8 Two-dimensional energy spectrum with different filtering techniques the turbulent kinetic energy and results in negative bias at both low and high frequency ranges of the energy spectrum. Combination of the Weiner filter and the median filter corrects the bias in the low frequency range, caused by the application of median filter; however, the negative bias at the high frequency range still exists. Thus, there is limited improvement on the reduction of energy spectrum by combining Wiener filter and median filter.

The percentage filtering errors (\%) of the global mean velocity and rms of the velocity fluctuations and the ratio of the rms of the velocity fluctuations in $\mathrm{x}$ and $\mathrm{y}$ directions are shown in Table 3. The median filter replaces the current pixel entry with the median value of the neighboring pixel entries within the square filter window size of $n \times n$. As a result, the error percentage of the rms of velocity fluctuations increases from $2.0 \%$ to a maximum of $17.9 \%$, when the filter window size varies from 2 to 5 pixels, respectively. The effect of median filter on the ratio of the rms of velocity fluctuations is limited within $0.7 \%$, suggesting good reproduction of global homogeneity. The filtering variation in the global mean velocity ranges from 4.3 to $15.2 \%$. However, the absolute value of the global mean velocity decreases towards zero. Thus, the median filter introduces limited effect on the global zero mean velocity and global homogeneity. There is a notable reduction in the turbulent kinetic energy up to $30 \%$ with the maximum error in rms of velocity fluctuations of $17.9 \%$ in the case of median filter of $5 \times 5$ vector spacing.

In addition, the global homogeneity is examined as the ratio of the average of the two eigenvalues of the rate-ofstrain tensor calculated from the raw and filtered velocity fields. The percentage filtering error on the ratios of the two eigenvalues are summarized in Table 4. The filtering process with the median and Wiener filters has slight influence on the global homogeneity with the ratio of two eigenvalues slightly lower than unity and is considered to be within a reasonable range with a maximum deviation of $2.6 \%$. This is in agreement with the observation obtained from the percentage error in the ratio of the rms of the velocity fluctuations, while the eigenvalues of the rate-of-strain tensor are

Table 3 Percentage filtering error (\%) on turbulent statistics

\begin{tabular}{llllrr}
\hline Filter error $(\%)$ & Raw exp & $\begin{array}{l}\text { Exp + median } 2 \times 2 \\
\text { vector spacing }\end{array}$ & $\begin{array}{l}\text { Exp + median } 3 \times 3 \\
\text { vector spacing }\end{array}$ & $\begin{array}{l}\text { Exp + median } 5 \times 5 \\
\text { vector spacing }\end{array}$ & $\begin{array}{l}\text { Exp }+ \text { median } 5 \times 5 \\
\text { vector spacing }+ \\
\text { Wiener }\end{array}$ \\
\hline$\left\langle\tilde{u}_{1}\right\rangle$ & 9.9 & 6.8 & 6.0 & 4.3 & 1.6 \\
$\left\langle\tilde{u}_{2}\right\rangle$ & 9.9 & 6.9 & 9.8 & 15.2 & 3.2 \\
$\left\langle\tilde{u}_{1, \mathrm{rms}}\right\rangle$ & 25.8 & 2.1 & 7.9 & 17.4 & 13.6 \\
$\left\langle\tilde{u}_{2, \mathrm{rms}}\right\rangle$ & 26.6 & 2.0 & 8.2 & 17.9 & 13.8 \\
$\left\langle\tilde{u}_{1, \mathrm{rms}}\right\rangle /\left\langle\tilde{u}_{2, \mathrm{rms}}\right\rangle$ & 0.6 & 0.03 & 0.3 & 0.7 & 0.3 \\
\hline
\end{tabular}


Table 4 Percentage filtering error $(\%)$ on the ratio of the average of the two eigenvalues of the rate-of-strain

\begin{tabular}{llllll}
\hline $\begin{array}{l}\text { Percentage filter- } \\
\text { ing error }(\%) \text { on } \\
\text { the ratio of the } \\
\text { two eigenvalues }\end{array}$ & Raw exp & $\begin{array}{l}\text { Exp + median } \\
2 \times 2 \text { vector } \\
\text { spacing }\end{array}$ & $\begin{array}{l}\text { Exp + median } \\
3 \times 3 \text { vector } \\
\text { spacing }\end{array}$ & $\begin{array}{l}\text { Exp + median } \\
5 \times 5 \text { vector } \\
\text { spcaing }\end{array}$ & $\begin{array}{l}\text { Exp + median } \\
5 \times 5 \text { vector spac- } \\
\text { ing + Wiener }\end{array}$ \\
\hline$\left\langle\tilde{\Lambda}_{1}\right\rangle /\left\langle\tilde{\Lambda}_{2}\right\rangle$ & 0.4 & 1.2 & 1.3 & 2.6 & 0.2 \\
\hline
\end{tabular}

more prone to the filtering techniques with slightly higher error percentage.

Thus, by the velocity vector field restored by the Wiener filter as the benchmark, all of the 4 proposed digital filtering techniques (median filter $2 \times 2,3 \times 3,5 \times 5$ vector spacing and linear combination of Wiener filter and median filter $5 \times 5$ vector spacing) are capable of recovering the rate-of-strain tensor and topological characteristics derived from velocity invariants with negligible modification on the turbulence global homogeneity and zero mean velocity. The negative bias on the high frequency range of the energy spectrum is improved with the addition of the Wiener filter. However, the reduction in the turbulent kinetic energy is still up to $20-30 \%$. In addition, there are limitations of the current proposed filtering technique, since the shape of the joint pdfs of velocity invariants and pdfs of eigenvalues of rate-of-strain tensor could not be fully 'recovered' to coincide with the joint pdfs of un-blurred, noise-free velocity vector invariants from DNS data. This is possibly due to the fact that the coupling of Gaussian noise and Salt and Pepper noise does not follow a linear relationship, as modeled in Eq. (14), thus the digital filtering techniques needed are not linear operators. To fully resolve the noise elimination in evaluating topological characteristics of turbulent flows requires constructing a non-linear noise model and modifying further the digital filtering techniques. However, this is beyond the content of the current work. The effects of digital filtering techniques on the statistical quantifications of the turbulent velocity stagnation points are evaluated in the following Section to establish the appropriate digital filtering method in identifying turbulent topological characteristics.

\section{On the clustering of 'zero velocity points'}

It has been found in the previous sections that the median filter is an effective method to remove the Salt and Pepper noise, which is the dominant factor in the evaluation of the topological characteristics of the turbulent flow. To demonstrate the influence of median filtering of the PIV measured velocity data on the identified topological turbulent zero velocity points, an instantaneous spatial distribution of zero velocity points in the flow field, identified with different median filter window sizes, is shown in Fig. 9.
Fig. 9 Instantaneous spatial distribution of zero velocity points identified with different Median filter window size. a Raw PIV data; b PIV velocity field filtered by $2 \times 2$ vector spacing window sized median filter; c PIV velocity field filtered by $3 \times 3$ vector spacing window sized median filter; $\mathbf{d}$ PIV velocity field filtered by $5 \times 5$ vector spacing window sized median filter; e noise-free DNS data

Detailed flow structures can be observed after filtering the PIV velocity with a $2 \times 2$ vector spacing median filter. The morphology of the zero velocity points remains and the main structures are captured after filtering with $3 \times 3$ and $5 \times 5$ vector spacing median filters. It is also observed that the representation of zero velocity points with $3 \times 3$ and $5 \times 5$ vector spacing Median filter are similar to that of the noise-free DNS data, forming clearedged clusters. Since these are instantaneous spatial distributions, the results from the experiments and the DNS data could not be identical. The statements rely on visual inspection of Fig. 9, which demonstrates the presence of spatial clustering of the zero velocity points in the experiments and the DNS data. Quantitative description of the clustering of the zero velocity points follows.

The saddle points, one of the turbulent topological flow patterns, are of interest in the study of droplet-laden flows, since they are possibly responsible for the preferential concentration of dispersed particles (Chen et al. 2006; Goto and Vassilicos 2006, 2008). The acceleration field is estimated with the back Eulerian method from two velocity fields, measured with PIV. Thus, the influence of the noise elimination digital filtering technique is examined on the clustering behavior of the saddle points of velocity vector fields, also referred to as zero velocity points. In the following discussion, zero velocity points are used to represent the saddle points of the velocity vector fields. The clustering behavior of the zero velocity points is quantified by two methods, namely the Radial Distribution Function (RDF) (Sundaram and Collins 1997) and Voronoï analysis (Monchaux et al. 2010).

The radial distribution function (RDF) was proposed by Sundaram and Collins (1997) and defined as:

$\mathrm{RDF}=\frac{N\left(r_{i}\right) A}{A\left(r_{i}\right) N}$, 


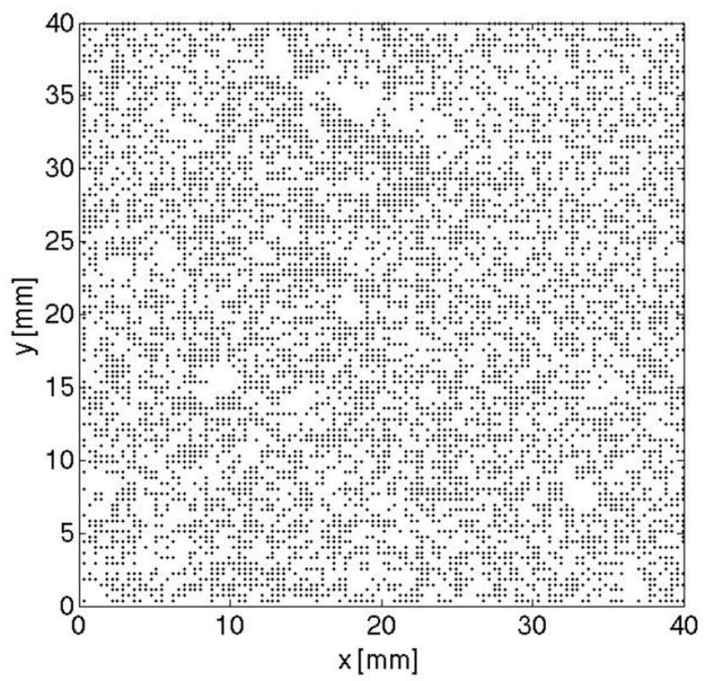

(a)

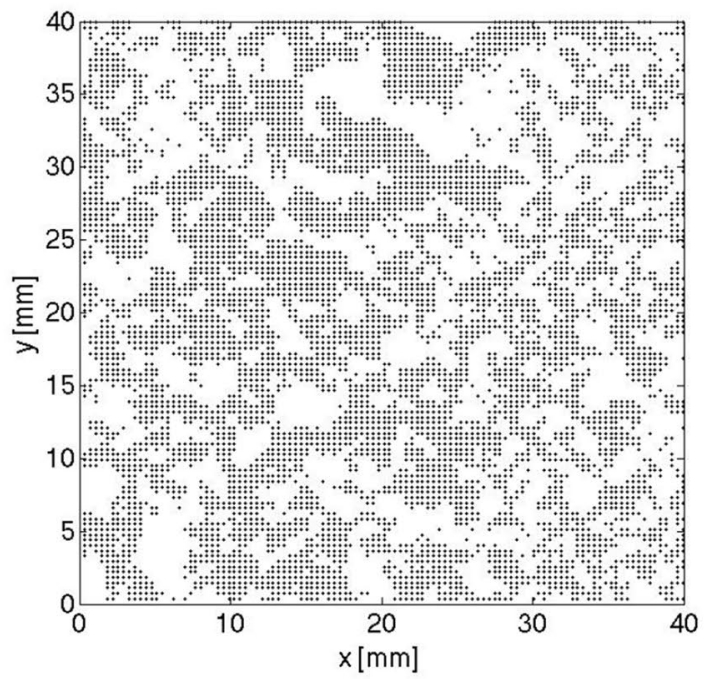

(c)

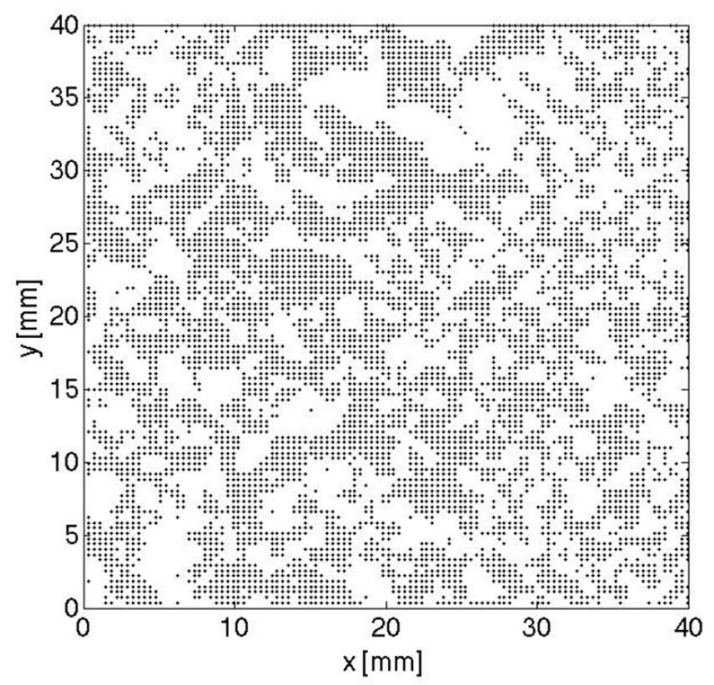

(b)

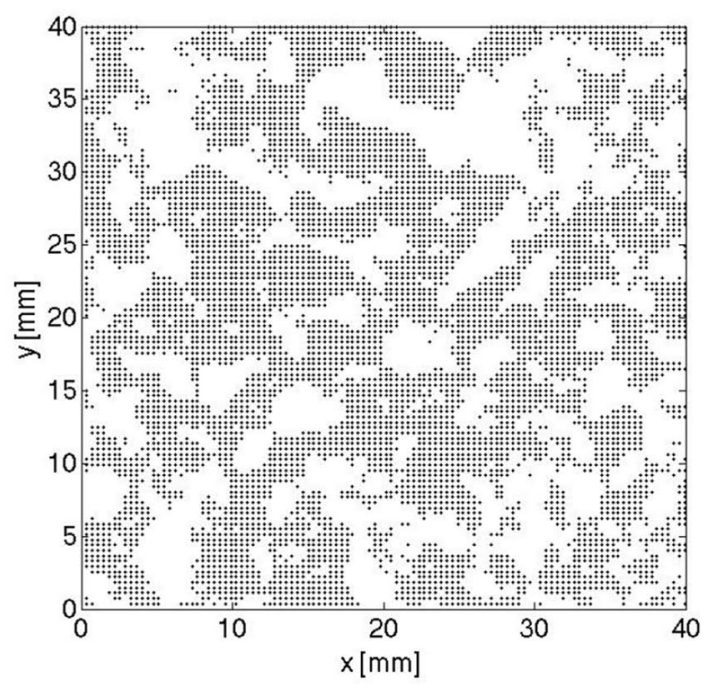

(d)

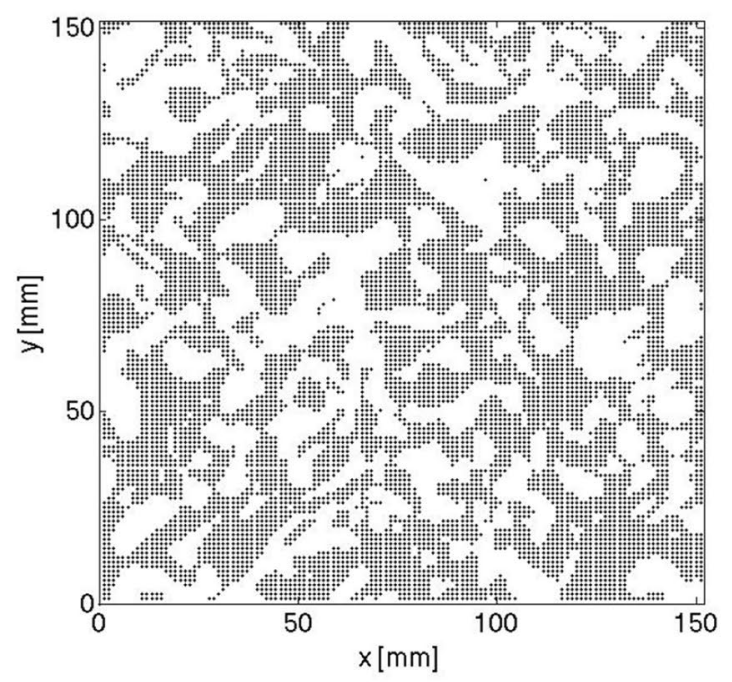

(e) 


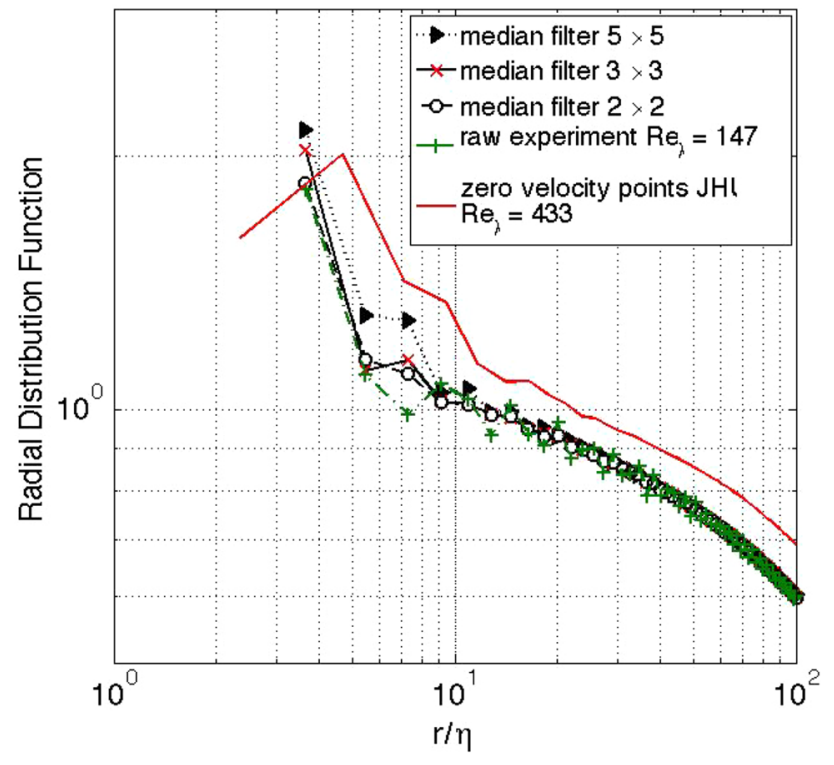

(a)

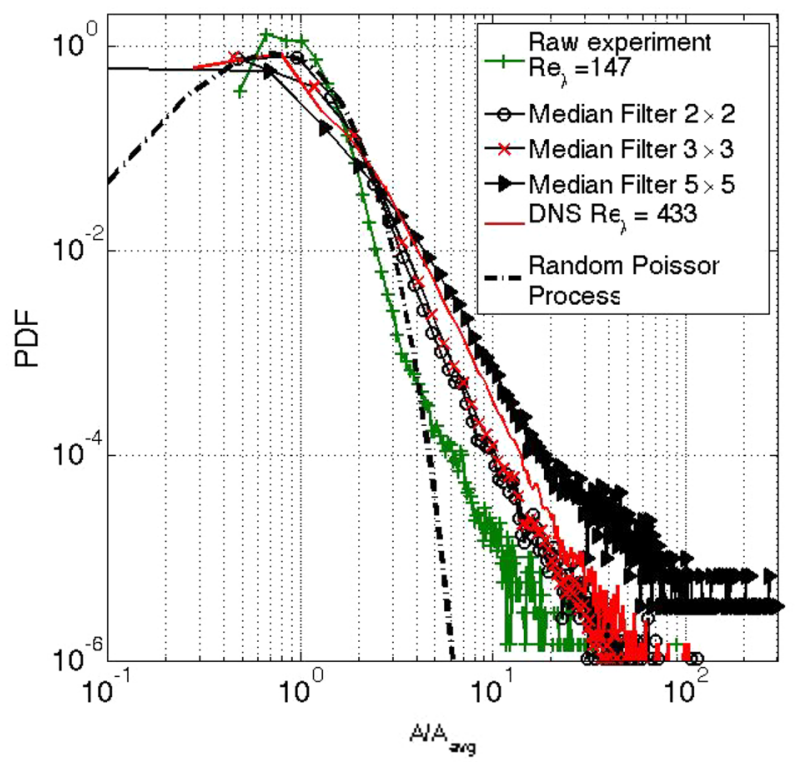

(b)

Fig. 10 Quantification of instantaneous clustering of turbulent zero velocity points. a Radial distribution function (RDF); b probability distribution of the normalized Voronoï area

where $N\left(r_{i}\right)$ is the number of zero velocity points in a ring with width of $2 d r$ and radius $r_{i}$ from the center of a randomly selected location. $A\left(r_{i}\right)$ is the area of the ring between $r-d r$ to $r+d r, A$ is the total area and $N$ is the total number of zero velocity points on an image of the flow field. The RDF measures the number density of the zero velocity points within a ring of certain radius relative to the average zero velocity points number density in

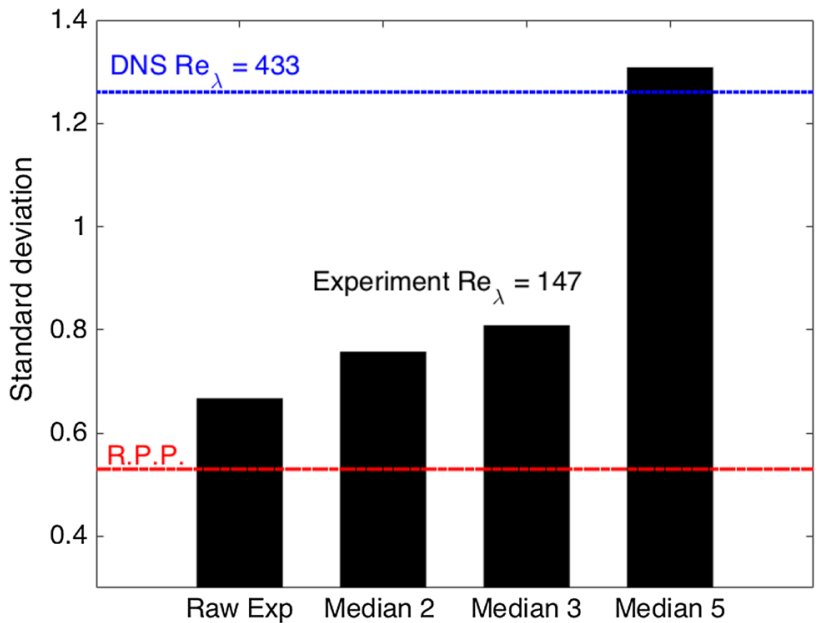

Fig. 11 The standard deviation of the normalized Voronoï area

the area of interest. As a consequence, RDF values larger than 1 indicate clustering above the average number density across the area of interest.

Figure 10a shows the RDF of zero velocity points derived from raw, median filtered experimental data and noise-free DNS data. All results demonstrate that there is strong clustering of zero velocity points, since the value of RDF is larger than 1 . The lengthscale of the clusters is identified when the value of RDF becomes 1 . It can be seen from Fig. 10a that the lengthscale of the clusters is around 10-15 times the Kolmogorov scale $\eta$ for the experimental results, while the DNS results indicate values, which are around $20 \eta$.

It is expected that the data filtered by Median filter with $5 \times 5$ vector spacing window size are measured with the highest clustering intensity and the ones from the raw experimental data return the least degree of clustering, agreeing with the visualization of Fig. 9. This is illustrated in Fig. 10a, where the dotted RDF curve marked with triangular symbols, linked to the data filtered by $5 \times 5 \mathrm{vec}-$ tor spacing Median Filter, is slightly above the other three curves of the data filtered by $3 \times 3$ vector spacing Median Filter, the data filtered by $2 \times 2$ vector spacing Median filter and the raw experimental data for cluster lengthscales smaller than around $10 \eta$. Also, it is interesting that there is a fairly good spatial correlation for cluster lengthscales larger than $10 \eta$ between all the RDFs regardless of the data noise level. It seems that, although the application of the Median filter significantly alters the shape of the joint pdfs of the velocity invariants, the RDF measurements of the clustering of the turbulent zero velocity points are not affected much by the applied Median Filter. The magnitude of the RDF of the noise-free DNS data is higher than 
the experimental result, indicating stronger clustering of zero velocity points occurring in the numerical simulation. However, the DNS and experimental RDF have the same behavior (i.e., slope) across all the scales. The remaining differences between DNS and experiments may be due to the higher turbulent intensity represented by a turbulent Reynolds number of $R e_{\lambda}=433$ compared to the considered experimental condition of $R e_{\lambda}=147$. It is noted that the physical mechanisms leading to the clustering of turbulent 'zero velocity' points is not the focus of the current paper, but was discussed by Lian (2014).

The Voronoï analysis decomposes the two-dimensional space into individual Voronoï cells that correspond to each zero velocity point, where each cell surface is closer to the considered zero velocity point than any other points. The Voronoï cell area $A$ is the inverse of the local concentration of zero velocity points. Thus, the distribution function of Voronoï cell areas can provide information of the local clustering level of the zero velocity points. The probability distribution function of the normalized Voronoï cell areas $A / A_{\text {avg }}$ has been applied to the quantification of clustering (preferential concentration) of particles (Monchaux et al. 2010).

Voronoï analysis has been applied here to evaluate the clustering of the zero velocity points in the measured instantaneous turbulent flow fields in the 'box of turbulence'. The normalized pdfs of the Voronoï areas surrounding zero velocity points derived from raw and median filtered data are shown in Fig. 10b. It should be noted that the normalized Voronoï area pdfs are more sensitive to the denoising process in comparison to the robustness observed in the RDF analysis. The application of the Median filter with $2 \times 2,3 \times 3$ and $5 \times 5$ vector spacing window size results in a stronger degree of clustering compared to the raw measurements, by the pdfs of Voronoi areas showing the larger deviation from the random poisson process (RPP) of Voronoi areas (Monchaux et al. 2010). The zero velocity points of the raw experimental data are more likely to be distributed randomly, as expected due to the noise on the image. The degree of clustering correlates with the increased window size of Median Filter. The observed increase in the degree of clustering could possibly be due to the fact that the Voronoï analysis is more accurate in capturing the clustering statistics of zero velocity points, since the averaging occurs once per image. It is consistent with both the visual inspection of Fig. 9 and the results for the RDF of Fig. 10a that the noise-free DNS data and the experimental data filtered by Median filter with $5 \times 5$ vector spacing window size show a stronger degree of clustering. Generally, for scales lower than around $10 \eta$, there is good agreement between the pdfs of the Voronoi areas from DNS and experimental data. Deviations occur at the larger scales, where still the results from experimental data processed with Median filter with window $3 \times 3$ vector spacing remain closer to the quantification results. Increasing the window size to $5 \times 5$ vector spacing result in the magnitude of clustering higher than that of the noise-free DNS data, indicating stronger clustering of zero velocity points, in contrary to the RDF quantification.

The standard deviation of the normalized Voronoï area is a single metric representing the magnitude of clustering (Monchaux et al. 2010), with the value of the random poisson process as 0.53 . Larger value of the standard deviation suggests stronger degree of clustering. The comparison between raw and filtered experiment as well as DNS is shown in Fig. 11, which supports the findings in Fig. 10b that applying median filter increases the magnitude of clustering. The median filter with window of $5 \times 5$ vector spacing increases the magnitude above the DNS at $\operatorname{Re}_{\lambda}=433$. Thus, the window size of the Median filter is to be limited to $3 \times 3$ vector spacing, which is effective to eliminate the experimental noise to a satisfactory level and extract the two-dimensional topological turbulent flow patterns without altering its statistical quantifications. It should be noted that the normalized Voronoï area pdfs of zero velocity points are calculated based on the PIV vector field with vector spacing of 8 pixels, effectively $364 \mu \mathrm{m}$, and improvement of the spatial resolution of the measurements could improve the accuracy to the extracted flow patterns.

To summarize, the clustering of instantaneous turbulent 'zero velocity' points, derived from raw and median filtered experimental data, is quantified by RDF and Voronoï analysis. For the quantifications with RDF, it seems that the RDF measurements of the clustering of the turbulent 'zero velocity' points are not affected much by the applied median filter on the images, although the application of the Median filter significantly alters the shape of the joint pdfs of the velocity invariants. Also, it is interesting that there is a fairly good spatial correlation for cluster lengthscales larger than $10 \eta$ between all the RDFs regardless of the filtering process. However, for the quantification with Voronoï analysis, the clustering behavior of the turbulent zero velocity points is found to be sensitive to the filtering techniques. There is no significant difference in terms of clustering magnitude compared to other data sets to distinguish the appropriate Median filter window size. For consistency in quantifying the magnitude of clustering with RDF and Voronoi analysis, the velocity field processed by Median filter with $3 \times 3$ vector spacing window size, is considered the most appropriate for noise elimination in evaluating turbulent topological flow patterns. Therefore, the current analysis of the clustering behavior of zero velocity points can be used to evaluate suggestions that the clustering behavior of dispersed droplets by the flow turbulence is correlated 
with the turbulent topological points (Chen et al. 2006; Goto and Vassilicos 2006, 2008). This study is described in Lian (2014).

\section{Conclusions}

This paper evaluates a data processing approach to calculate the topological characteristics of turbulent flow structures in homogeneous, isotropic turbulence without mean flow from velocity data measured by two-dimensional TRPIV. To identify the primary noise types presented in the experimental data, we added noise to the velocity field, according to Eq. (14), to noise-free numerical data from the JHU open source database. We found the two quantities are sensitive to two types of noise. The tensor invariants seem to be more sensitive to the Salt and Pepper noise, while the principle components to Gaussian noise. So the median filter and the Wiener filter were chosen to eliminate the Salt and Pepper noise and Gaussian noise, respectively. We evaluated the effects of both filters on the tensor invariants and principle components obtained from experiments following the optimized noise filtering processes identified from the numerical data. The $u_{\mathrm{rms}}$ and other flow quantities are further analyzed to quantify the errors on the global flow kinetic energy level, homogeneity and isotropy due to the filtering process. Once satisfied with the induced filtering errors, the statistical clustering behavior of the zero velocity points was evaluated to conclude the optimisation of the filtering method (a median filter with window size $3 \times 3$ vector spacing) to extract zero velocity points from the TR-PIV measurements. The evaluation of the effects of noise through the DNS data set and the optimization of the digital filters allowed us to extend the digital filtering to additional quantities related to the experimental data. The main findings of this paper are summarized as follows.

1. A 'noise' model is established by adding Gaussian noise and Salt and Pepper noise to the velocity vector fields of the DNS velocity data of homogeneous and isotropic turbulence from the JHU database. The digital Wiener Filter and the median filter were applied to eliminate experimental noise. The Salt and Pepper noise is found to be the main noise source in the evaluation of velocity invariants and topological characteristics of turbulent flows. The Gaussian noise is found to be the dominating noise source influencing the estimate of the rate-of-strain tensor.

2. With the velocity vector field restored by the Wiener filter used as the benchmark, the 4 proposed digital filtering techniques (median filter $2 \times 2,3 \times 3,5 \times 5$ vector spacing and linear combination of Wiener filter and median filter $5 \times 5$ vector spacing) can recover the rateof-strain tensor and topological characteristics derived from velocity invariants with negligible modification on the turbulence global homogeneity and zero mean velocity. However, the application of the digital filtering techniques causes reduction in the turbulent kinetic energy.

3. The clustering characteristics of the instantaneous turbulent 'zero velocity' points are quantified by RDF and Voronoï analysis. It was found that the RDF measurements of the clustering of the turbulent velocity saddle points are not affected much by the applied median filtering, while the Median filter de-noising process results in noticeable difference in the normalized Voronoï area pdfs, when the window size of the median filter exceeds $3 \times 3$ vector spacing. Thus, $3 \times 3$ median filter is found to be the appropriate filtering method of the measured velocity images to extract the statistical turbulent topological characteristics.

Acknowledgements Financial support from the Engineering and Physical Sciences Research Council (EPSRC) under Grant EP/ E029515/1 is acknowledged. H. Lian was supported by the Chinese Scholarship Council (CSC).

Open Access This article is distributed under the terms of the Creative Commons Attribution 4.0 International License (http:// creativecommons.org/licenses/by/4.0/), which permits unrestricted use, distribution, and reproduction in any medium, provided you give appropriate credit to the original author(s) and the source, provide a link to the Creative Commons license, and indicate if changes were made.

\section{References}

Antonia R, Zhu Y, Kim J (1994) Corrections for spatial velocity derivatives in a turbulent shear flow. Exp Fluids 16:411-413

Atkinson C, Buchmann NA, Soria OA (2014) On the appropriate filtering of PIV measurements of turbulent shear flows. Exp Fluids 55:1654

Bragg AD, Ireland PJ, Collins LR (2015) On the relationship between the non-local clustering mechanism and preferential concentration. J Fluid Mech 780:327-343

Buxton O, Laizet S, Ganapathisubramani B (2011) The effects of resolution and noise on kinematic features of fine-scale turbulence. Exp Fluids 51:1417-1437

Cardesa J, Mistry D, Gan L, Dawson J (2013) Invariants of the reduced velocity gradient tensor in turbulent flows. J Fluid Mech 716:597-615

Charalampous G, Hardalupas Y (2010) Clustering of mono-disperse and poly-disperse particles in a "box of turbulence". In: Proceedings of 7th ICMF 2010, Tampa, FL USA

Chen L, Goto S, Vassilicos JC (2006) Turbulent clustering of stagnation points and inertial particles. J Fluid Mech 553:143-154

Christensen K (2004) The influence of peak-locking errors on turbulence statistics computed from PIV ensembles. Exp Fluids 36(3):484-497 
Eaton JK, Fessler JR (1994) Preferential concentration of particles by turbulence. Int J Multiph Flow 20:169-209

George WK (2013) Lectures in turbulence for the 21st century

Goepfert C, Marié JL, Chareyron D, Lance M (2009) Characterization of a system generating a homogeneous isotropic turbulence field by free synthetic jets. Exp Fluids 48:809-822

Goto S, Vassilicos JC (2006) Self-similar clustering of inertial particles and zero acceleration points in fully developed two-dimensional turbulence. Phys Fluids 18:115103

Goto S, Vassilicos JC (2008) Sweep-stick mechanism of heavy particle clustering in fluid turbulence. Phys Rev Lett 100:054503

Hardalupas Y, Taylor AMKP, Whitelaw JH (1990) Velocity and size characteristics of swirling liquid-fuelled flames. Proc R Soc Lond A 428:129-155

Hardalupas Y, Taylor AMKP, Whitelaw JH (1992) Particle dispersion in a vertical round sudden expansion flow. Phil Trans R Soc Lond A 341:411-442

Huang TS, Yang GJ, Tang GY (1979) Fast two-dimensional median filtering algorithm. IEEE Trans Acoust Speech Signal Process $1: 13-18$

Hwang W, Eaton JK (2004) Creating homogeneous and isotropic turbulence without a mean flow. Exp Fluids 36:444-454

Khashehchi M, Elsinga GE, Ooi A, Soria J, Marusic I (2010) Studying invariants of the velocity gradient tensor of a round turbulent jet across the turbulent/nonturbulent interface using Tomo-PIV. In: 15th Int Symp on Applications of Laser Techniques to Fluid Mechanics Lisbon, Portugal, 05-08 July, 2010

Lavoie P, Avallone G, Gregorio FD, Romano GP, Antonia RA (2007) Spatial resolution of PIV for the measurement of turbulence. Exp Fluids 43:39-51

Li Y, Perlman E, Wan M, Yang Y, Meneveau C, Burns R, Chen S, Szalay A, Eyink G (2008) A public turbulence database cluster and applications to study lagrangian evolution of velocity increments in turbulence. J Turbul 9:31-61

Lian H (2014) Droplet dispersion in homogeneous and isotropic turbulence. PhD thesis, Imperial College London

Lian H, Charalampous G, Hardalupas Y (2013) Preferential concentration of poly-dispersed droplets in stationary isotropic turbulence. Exp Fluids 54:1525

Maxey MR (1987) The gravitational settling of aerosol particles in homogeneous turbulence and random flow field. J Fluid Mech 174:441-465

Monchaux R, Bourgoin M, Cartellier A (2010) Preferential concentration of heavy particles: a voronoi analysis. Phys Fluids 22(10): 103304
Okamoto K, Nishio S, Saga T, Kobayashi T (2000) Standard images for particle-image velocimetry. Meas Sci Technol 11:685-691

Oxlade AR, Valente PC, Ganapathisubramani B, Morrison JF (2012) Denoising of time-resolved PIV for accurate measurement of turbulence spectra and reduced error in derivatives. Exp Fluids 53:1561-1575

Perlman E, Burns R, Li Y, Meneveau C (2007) Data exploration of turbulence simulations using a database cluster. In: Proceedings of the 2007 ACM/IEEE Conference on Supercomputing, SC'07, vol 23, pp 1-11

Perry A, Chong MA (1987) Description of eddying motions and flow patterns using critical-point concepts. Annu Rev Fluid Mech 19:125-155

Petrou M, Petrou C (1999) Image processing: the fundamentals. Wiley, New York

Press W, Teukolsky S, Vetterling W, Flannery B (1988) Numerical recipes in $\mathrm{C}$ : the art of scientific computing. Cambridge University Press, Cambridge

Soulopoulos N, Hardalupas Y, Taylor AMKP (2014) Scalar dissipation rate measurements in a starting jet. Exp Fluids 55:1685

Sugii Y, Nishio S, Okuno T, Okamoto K (2000) A highly accurate iterative PIV technique using gradient method. Meas Sci Technol 11:1666-1673

Sundaram S, Collins LR (1997) Collision statistics in an isotropic particle-laden turbulent suspension. Part 1. Direct numeric simulation. J Fluid Mech 335:75-109

Vétel J, Garon A, Pelletier D (2011) Denoising methods for time resolved PIV measurements. Exp Fluids 51:893-916

Wang LP, Maxey MR (1993) Settling velocity and concentration distribution of heavy particles in homogeneous isotropic turbulence. J Fluid Mech 256:27-68

Westerweel J (1994) Efficient detection of spurious vectors in particle image velocimetry data. Exp Fluids 16(3-4):236-247

Westerweel J (1997) Fundamentals of digital particle image velocimetry. Meas Sci Technol 8:1379-1392

Westerweel J (2000) Theoretical analysis of the measurement precision in particle image velocimetry. Exp Fluids 29:S003-S012

Worth N, Nickels T, Swaminathan NA (2010) Tomographic PIV resolution study based on homogeneous isotropic turbulence DNS data. Exp Fluids 49:637-656 